\title{
Internet of Things (IoT) based Smart Vehicle Security and Safety System
}

\author{
Yassine SABRI ${ }^{1}$ \\ Laboratory of Innovation in Management \\ and Engineering for Enterprise (LIMIE), \\ ISGA Rabat, 27 Avenue Oqba, \\ Agdal, Rabat, Morocco
}

\author{
Aouad Siham ${ }^{2}$ \\ Mohammed V University of Rabat \\ Smart Systems Laboratory (SSL) \\ ENSIAS, Morocco
}

\author{
Aberrahim Maizate ${ }^{3}$ \\ RITM- ESTC/CED -ENSEM, \\ University Hassan II Km7, \\ El jadida Street, B.P. \\ 8012, Oasis, Casablanca 8118
}

\begin{abstract}
The Internet of Things (IoT) is making human life easy in all aspects. The applications it offers are beyond comprehension. IoT is an abstract idea, a notion which interconnects all devices, tools, and gadgets over the Internet to enable these devices to communicate with one another. IoT finds application in various areas, such as intelligent cars and their safety, security, navigation, and efficient fuel consumption. This project puts forth a solution to achieve the desired outcome of saving precious human lives that are lost to road crashes .In this context, we propose to develop a system, we are designing and deploying a system that not only avoids accidents but also to take action accordingly. This research aims at dealing with the issues that cause fatal crashes and also integrates measures to ensure safety. Life without transportation is impossible to imagine; it makes far off places easy to reach and greatly reduces the travel time. But the problems which surface due to the ever-increasing number of vehicles on the road cannot be ignored. The project aims to eradicate a few of the major reasons of car crashes and also aims to integrate post-crash measures.
\end{abstract}

Keywords-Smart vehicle security; safety system; Internet of Things (IoT)

\section{INTRODUCTION}

Before the discovery of the wheel, primitive man would remain secluded from other groups and communities. They could commute only within walking distance. The discovery of the wheel entirely evolved the early man life. His social boundary also grew with time. With passing time, primitive man evolved to a mannered, civilized individual and refined the design of the wheel. With the advent of technology, transportation has become an indispensable part of our lives. Though it has countless advantages and uses, we have to deal with the major problem it brings with it that costs human life. Statistically, according to Ministry of Statistics and Programme Implementation, there were 114 million motor vehicles registered in India in the year 2009 and 159 million in the year 2012. The data provided by Delhi Statistical Hand Book clearly indicates the rise in the number of registered motor vehicles from 534,000 to 877,000 in the year 2014-2016 thus increasing the number of accidents and in turn the causalities associated with the surge. Data collected by the National Crime Bureau and Ministry of Road Transport and Highway revealed that in the year 2013 more than 100,000 people lost their lives in road rage. Despite the efforts of awareness campaigns, road signs, and traffic rules, motor accidents accounted for $83 \%$ of total traffic-related bereavements in the year 2015 as published by IndiaSpend.

\section{A. Motivation}

The Internet of Things (IoT) is making human life easy in all aspects. The applications it offers are beyond comprehension. IoT is an abstract idea, a notion which interconnects all devices, tools, and gadgets over the Internet to enable these devices to communicate with one another. It utilizes information technology, network technology, and embedded technology. Various sensors and tracking devices are coupled to deliver the desired outcome thus making lives easier. IoT finds application in various areas, such as intelligent cars and their safety, security, navigation, and efficient fuel consumption. This project puts forth a solution to achieve the desired outcome of saving precious human lives that are lost to road crashes. In the proposed system, we are designing and deploying a system that not only avoids accidents but also to take action accordingly.

\section{B. Aim of the Work}

This research aims at dealing with the issues that cause fatal crashes and also integrates measures to ensure safety. Life without transportation is impossible to imagine; it makes far off places easy to reach and greatly reduces the travel time. But the problems which surface due to the ever-increasing number of vehicles on the road cannot be ignored. The project aims to eradicate a few of the major reasons of car crashes and also aims to integrate post-crash measures. The reasons for automotive accidents focused here in this project are

- Nonchalant attitude towards the use of seat belts.

- Driving under the influence of alcohol.

- Distracted driving due to drowsiness.

The post-accident measure incorporated in the project is

- Intimation to the near and dear ones of the occurrence.

\section{Objectives}

The proposed project aims to achieve the following:

- Switch on the ignition only if the seat belts are locked in.

- Deploy a gas senor to make sure that driver is not drunk. If the driver is not drunk, only then will the engine ignite. 
- To ensure the driver is not drowsy, eye-blink sensors are deployed in the automobile.

- To circumvent a crash, a proximity sensor is deployed to discover the interruption in front of the automobile on the path.

- $\quad$ To ensure post-crash safety an alert system is deployed which makes use of a GPS system to attain the geographical location of the crashed vehicle and it is sent to a responsible and authorized individual. The accident is detected with the use of a vibration sensor.

\section{Paper Organization}

In the first section, an introduction has been provided to the whole project. All the fundamentals have been presented in which key modules of the project have been explained like the aims and objectives of the implemented system along with the motivation for choosing this project title. The second section of this project report reviews the literature surveys that have been performed to provide the basis for the implementation that is being performed. Equivalent and competing approaches that exist and have been worked upon are examined, recorded, and contrasted with the techniques and methods being implemented in this paper. These methods are further verified for any dichotomy that may be prevalent in their system. Further, methods are integrated to overcome those gaps. Starting from the third section, the technical aspect of the project is addressed. The basic framework and architecture of the methods are incorporated that will be realized in the building of smart vehicle safety and security systems. This is explained with the help of text and diagrams and flow charts. This helps in the step by-step visualization and organization of the project. The methodology of the project implementation is studied in greater depths in Section 4 . Point-by-point software and hardware constraints and requirements to be met to accomplish the obvious building guidelines are further enrolled and comprehended in detail. The final section comprises of the conclusions. The same has been used to supply the basis for the brief of what has been done and further work scope of the project is discussed to provide a summary.

\section{LiterATURE SURVEY}

\section{A. Survey of the Existing Models/Work}

For Pannu et al. [1], the emphasis is on making a monocular vision, self-sufficient auto model utilizing Raspberry $\mathrm{Pi}$ as a handling chip [6]. A high-definition camera alongside an ultrasonic sensor was utilized to give fundamental information from this present reality to the automobile. The automobile is $\mathrm{ft}$ for achieving the given goal securely and insight fully in this manner avoiding the danger of human mistakes. Numerous current calculations like path identification and impediment location are consolidated to give vital control to the auto. The paper undertakes the implementation of the system using Raspberry Pi, by the ethicalness of its processor. Kumar et al. [2] proposed the design and development of an accelerometer based system for driver safety. This framework is structured by using Raspberry Pi (ARM11) for quickly accessing the control and accelerometer for event discovery. If any event occurs the message is sent to the authorized personnel so they can take quick and immediate response to save the lives and abate the harms. The system only incorporates one module ignoring the other fatal causes thus making the proposed model incompetent and incomplete.

Sumit et al. [3] proposed a compelling strategy for the crash evasion arrangement of a vehicle to identify the hindrances present in the front and blind spot of the vehicle. The driver is alarmed with the help of a buzzer and an LED sign, as the distance between vehicle and obstacle reduces and is reflected on a display board. The ultrasonic sensor identifies the state of the object if it is moving or is stationary with respect to the vehicle. This system is valuable for discovering vehicles, bicycles, motorcycles, and pedestrians that cross by the lateral side of the automobile. The paper executes the proposed system using Raspberry $\mathrm{Pi}$ as the microcomputer but it limits out-of-the-box performance.

Mohamad et al. [4] proposed a proficient vehicle collision aversion framework inserted with an alcohol detector. This system has the capability of making the driver alert regarding the amount of alcohol consumed and depicting the same on an LCD screen. In addition it generates a warning using a buzzer to make the driver mindful of his or her own particular situation and to fag others in the encompassing zone [5]. The security segment proposed by this framework is the driver in an unusually abnormal state of tipsiness isn't allowed to drive an automobile as the start framework will be shut down. This method works in a way to intimidate the driver about his own condition, which is ironic because the person won't be mindful to take any action against it. The idea is novel but practically it is not workable.

\section{B. Summary/Gaps Identified in the Survey}

The current system showcases a mechanism for receiving the geographical coordinates of the automobile during a crash. This existent framework additionally provides a means of discovery of pre-crash with an object. But it does not target on the intensions that cause these fatal accidents. It does not focus on the crashes that are caused by drunk driving with the help of an alcohol/gas sensor and neither the negligence of use of seat belts.

Also these framework don't guarantee if the driver is wide awake or feeling drowsy. There is no use of eye-blink sensor for the same reason. Additionally, the current framework requires manual involvement. However, the proposed framework works on the shortcomings of the current work and is completely mechanized.

\section{OVERVIEW OF THE PROPOSED SYSTEM}

\section{A. Introduction and Related Concepts}

The proposed system utilizes an embedded system based on the Internet of Things and the Global System for Mobile Communication (GSM)[7] To avoid an accident, when the system is initiated, the seat belt is checked using a pressure sensor. If the driver is not wearing a seat belt, the engine is turned off. Then the alcohol sensor comes into play and checks for alcohol consumption, and if positive the engine is turned off. After these two main tasks [8], three things tiredness, collision, and obstacles - are checked using the eyeblink sensor, vibration sensor [9], and the infrared (IR) sensor 
[10], respectively. If there is a collision and the vibration sensor is active, then there is a message sent to the contact mentioned. If there is any obstacle present, then the buzzer beeps to tell the driver [11]. If the driver is feeling sleepy or drowsy, the eye blink sensor detects it and switches off the engine [12].

1) The system utilizes GSM technology for the communication of code pattern to transmit location coordinates.

2) The system is Arduino Uno based.

3) The system should be able to communicate even from physically far off distances.

4) The system uses an IR sensor[13], vibration sensor, alcohol sensor, eye-blink sensor, and pressure sensor [14].

5) To practically put together all the components and execute them, the composition of various sensing devices in our system is as shown in Figure 1

\section{B. Proposed System Model}

The software development system model that best suits this project and aligns itself with the needs of the given project is the Agile development model. Figure 2 is a block diagram, depicting the steps concerned in implementing the Agile development model.

In the Agile development model the entire requirement set is broken into numerous builds (Figure 3 and Figure 4). Various development stages take place here, making the development cycle a "multi-step waterfall" cycle. Cycles are split into tinier portions, making the modules easier to manage and implement. Every module goes through the planning, requirements analysis, design, implementation or building, and testing stages. A running version of the system is delivered at the end of the first iteration, so we get a working model early on during the product development cycle. Each iteration releases a model with added modules integrating more functions to the last release.

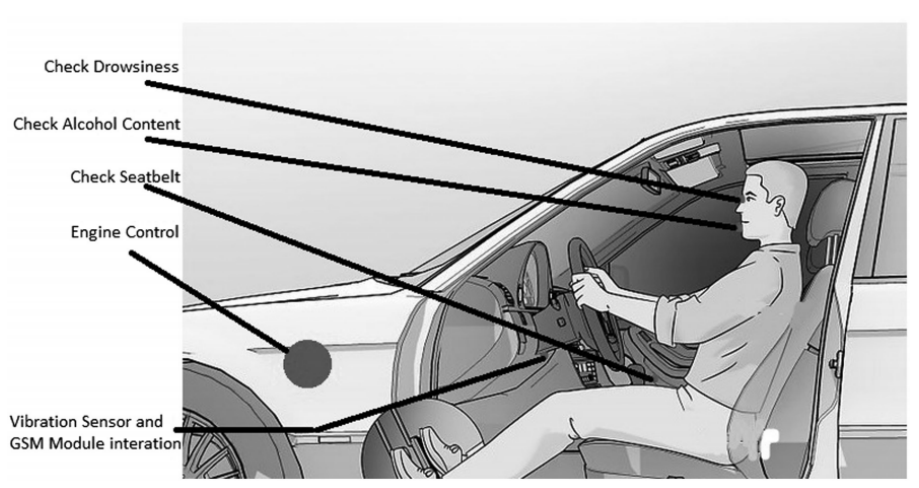

Fig. 1. System Design Implementation.

This process goes on until the complete system is developed. These iterations are repeated in a loop till an end version of the system is refined and is the expected outcome is obtained.

- As the project deploys a real-time checking and a monitoring system, the outputs produced are further used to take the necessary actions and are thus fed back to the code to give an appropriate action for the further events.

- This process is redundant and cyclic in nature which is implemented whenever a driver enters the automobile.

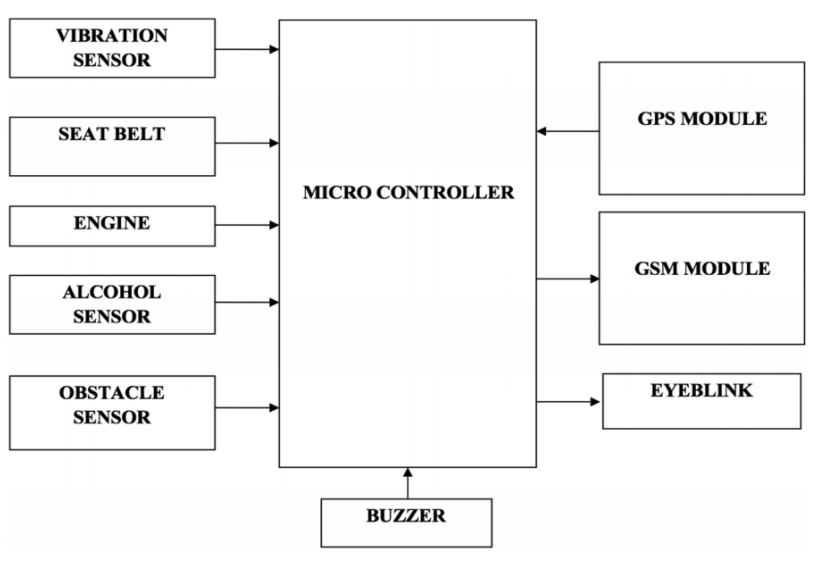

Fig. 2. Block Diagram

\section{Proposed System ANALYSIS AND DESIGN}

\section{A. Requirement Analysis}

The arrangement of the idea to implement the functional requirements is elucidated under the heading system design. The arrangement of the idea to implement the non-functional requirements is elucidated in the system architecture section of this project report. The imperative functional requirements of this project's objective accomplishment are:

1) The automotive system should have the capability to determine whether the seat belt is put on or not by the driver.

2) The implemented system should have the capability to determine whether alcohol has been consumed by the driver or not.

3) The automotive system should have the capability to determine the mental awareness of the driver in terms of if he is feeling sleepy.

4) The automotive system should have the capability to check whether the vehicle is not coming too close to the vehicle in front.

5) The automotive system should have the capability to determine whether an accident has already taken place and thus should have the capability of sending the location coordinates of accident to a responsible person with the help of GSM technology.

This project deals with problems which cause accidents and attempts to ensure safety. This project addresses various reasons that lead to fatal accidents. Roads are unpredictable and at every turn of the road can be fatal accidents present and one cannot rely on the driving sense of other drivers and the pedestrians. One needs to be self-aware of the environment and the vehicles around. The driver should take all the precautions and be mindful of the people on the road as well because every life has value. Common reasons for accidents are the 


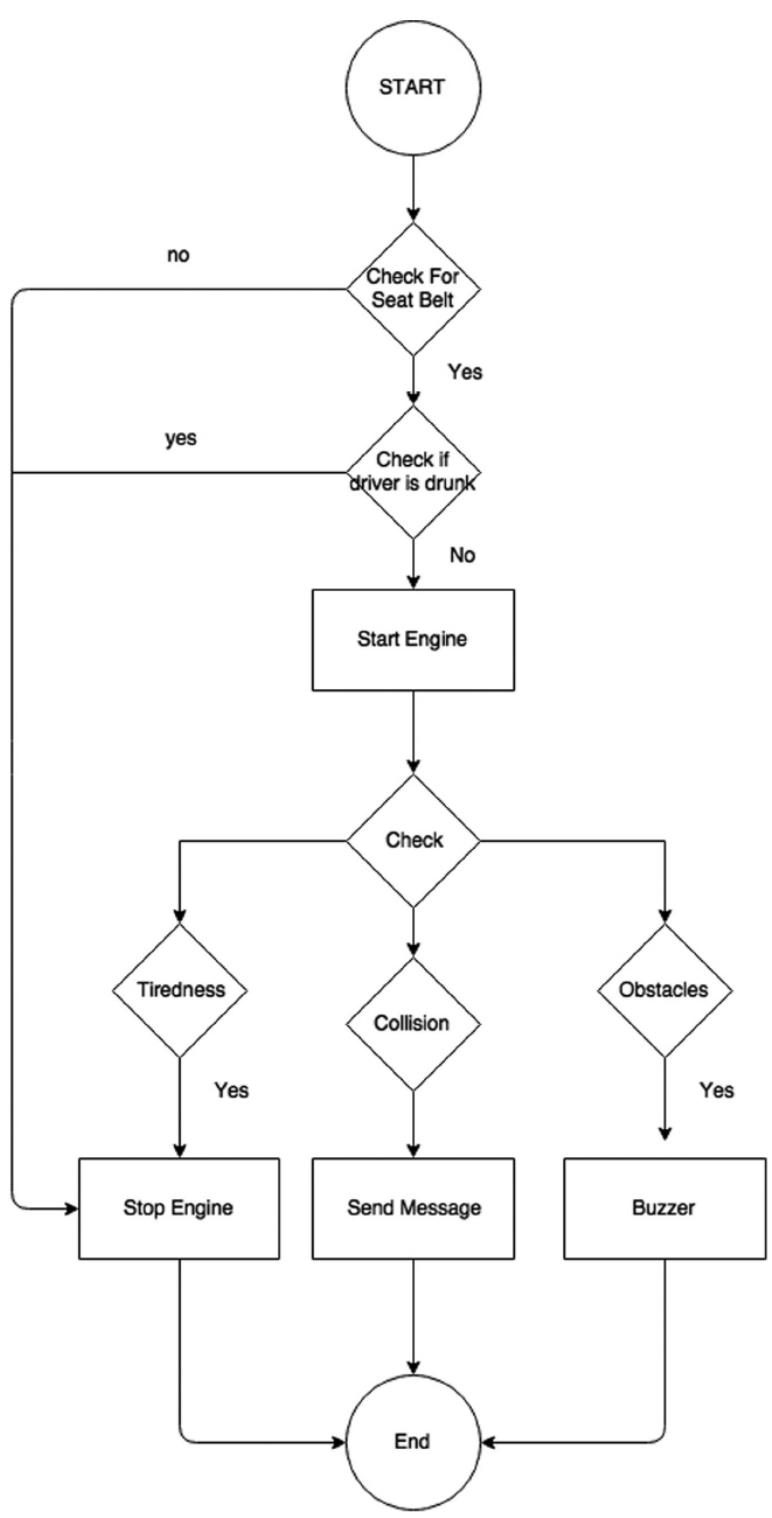

Fig. 3. UML Diagram (Activity Diagram).

lack of concentration of the driver on the road because of some distraction or because of lack of sleep of the driver. The product aims to provide the following functions:

- External forces should not result in the damaging of the system.

- The framework must be able to explicitly identify and discover problems related to the components.

- The issue detected should be reported back to the system.

The assumptions and dependencies are established in the beginning itself to give us a lucid understanding of the implementation of the product:

- Need of an appropriate GPS module to deliver exact geographical location coordinates.

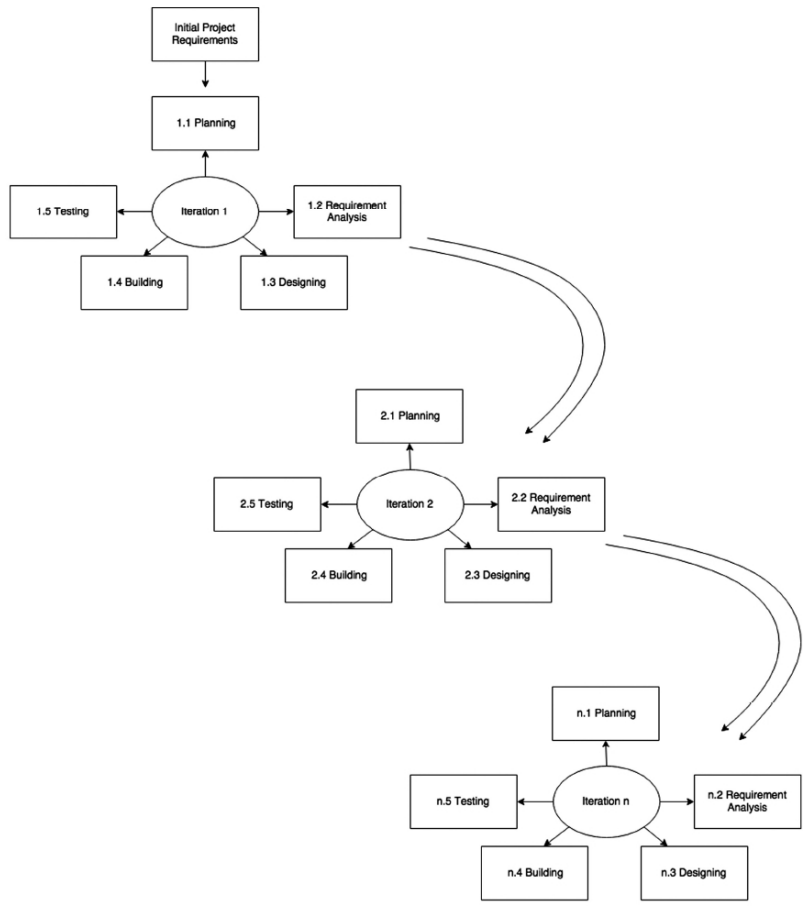

Fig. 4. Agile Model.

- The driver should be wearing the spectacles eye gear integrated with the eye-blink sensor.

- $\quad$ The system should always be connected to Internet.

- Proper placement of numerous proximity sensors can be added.

\section{B. System Requirements}

The output of the Smart Vehicle Security and Safety System is heavily dependent on Android application. The hardware components utilized for the project titled Smart Vehicle Security and Safety System are as follows. Arduino Uno board: The project utilizes Arduino Uno as the microcontroller. All the sensor components are attached and soldered to this microcontroller board and the microcontroller then takes the input and computes to give an appropriate output (Figure 5). Global vibration sensor: This project utilizes the vibration sensor to sense the accident and the crash of the automobile. This input received by the sensor is given

to the microcontroller Arduino Uno board that further utilizes the input to give a specific output (Figure 6). Alcohol/gas sensor: This project utilizes the alcohol sensor to sense the alcohol content in breath. This input received by the sensor is given to the microcontroller Arduino Uno board that further utilizes the input to give a specific output (Figure 7). Eye-blink sensor: This project utilizes the eye-blink sensor to sense the tiredness of the driver. This input received by the sensor is given to the microcontroller Arduino Uno board that further utilizes the input to give a specific output (Figure 8). Buzzer: This project utilizes the buzzer that signals and alerts the driver and the surroundings. The output is sent to the buzzer by the Arduino Uno board according to the computation (Figure 9). GPS module: This project utilizes 


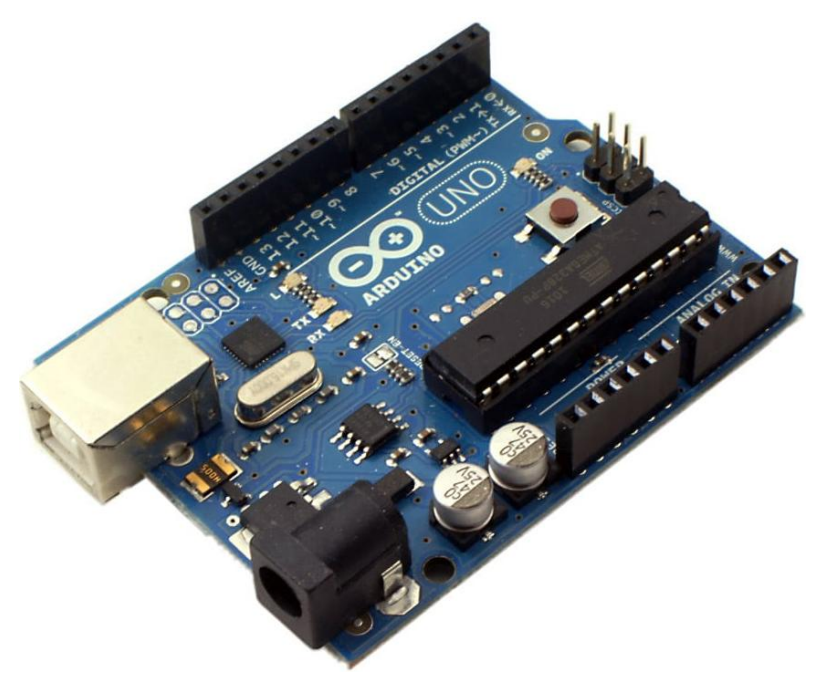

Fig. 5. Arduino Uno.

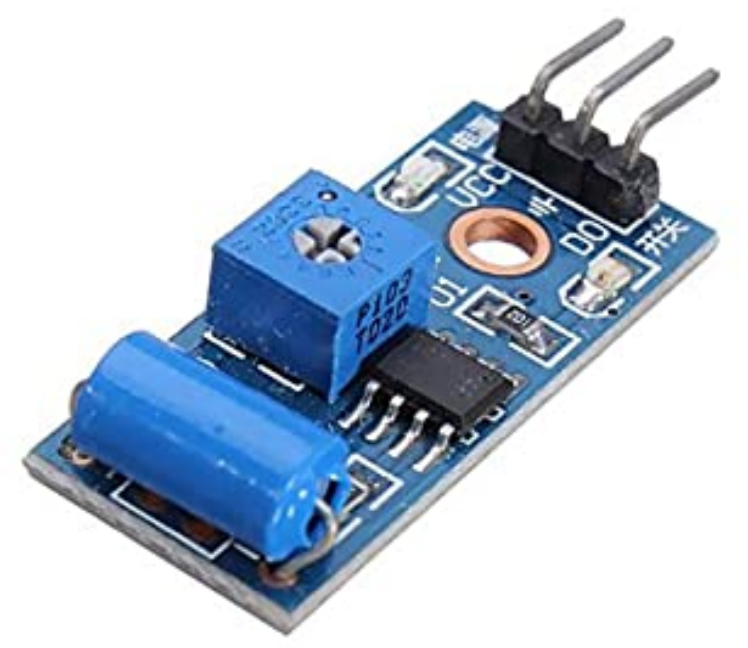

Fig. 6. Vibration sensor.

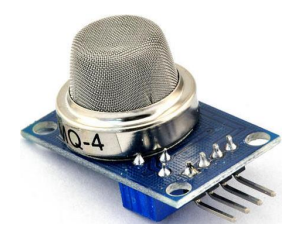

Fig. 7. Gas Sensor.

the GPS module to track the coordinates of the location of the where the project is present. This input received by the sensor is given to the microcontroller Arduino Uno board that further utilizes the input to give a specifc output (Figure 10). GSM module: This project utilizes the GSM module to communicate the coordinates of the location as detected by the GPS module. This input received by the sensor is given to

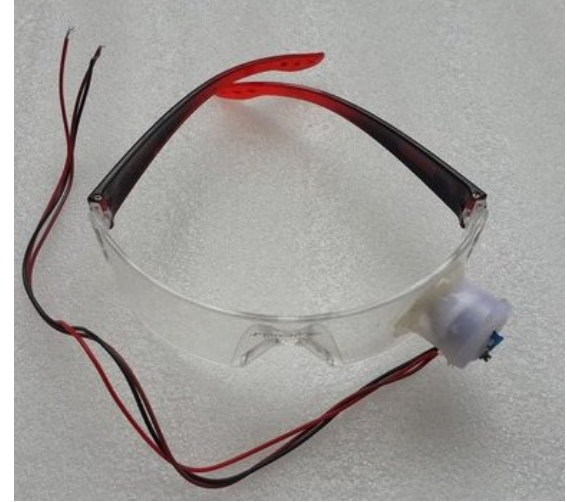

Fig. 8. Eye-blink Sensor.

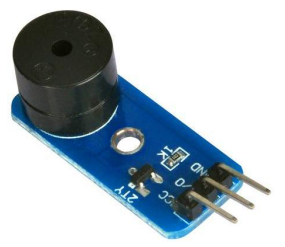

Fig. 9. Buzzer.

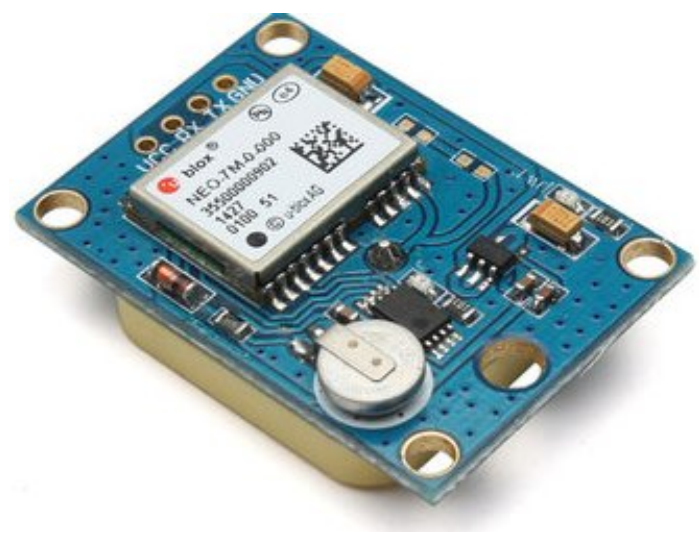

Fig. 10. GSM Module.

the microcontroller Arduino Uno board that further utilizes the input to give a specific output (Figure 10). This instructional exercise will disclose how to interface a GSM modem with Toradex modules (Figure 11).

\section{Results AND Discussion}

\section{A. Experimental Results}

The experimental results show that the proposed model gives us a better result as compared with other available devices. The output of the force sensitivity sensor is shown in Figure 12. This figure shows that the output provides more values as per the increase in time. Figure 13 shows the serial monitor of the vibration sensor.

Whereas, Figure 14 and Figure 15 display the percentage of crashes due to fatigue and causes of crashes, respectively. 


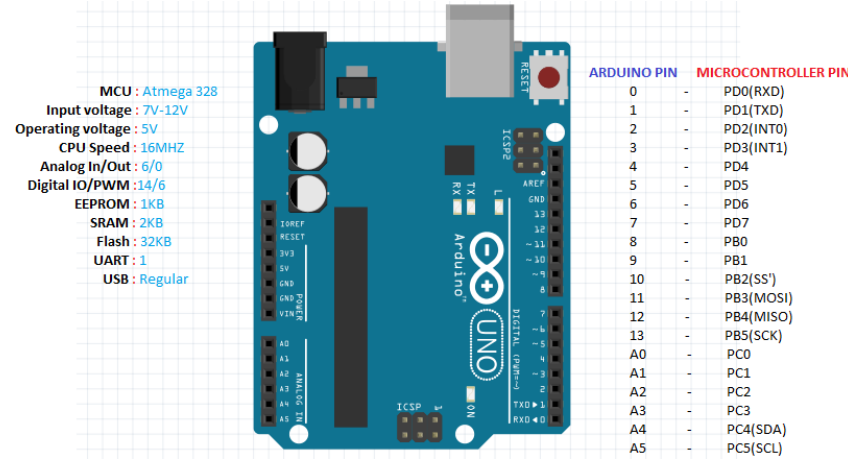

Fig. 11. Arduino Mapping.

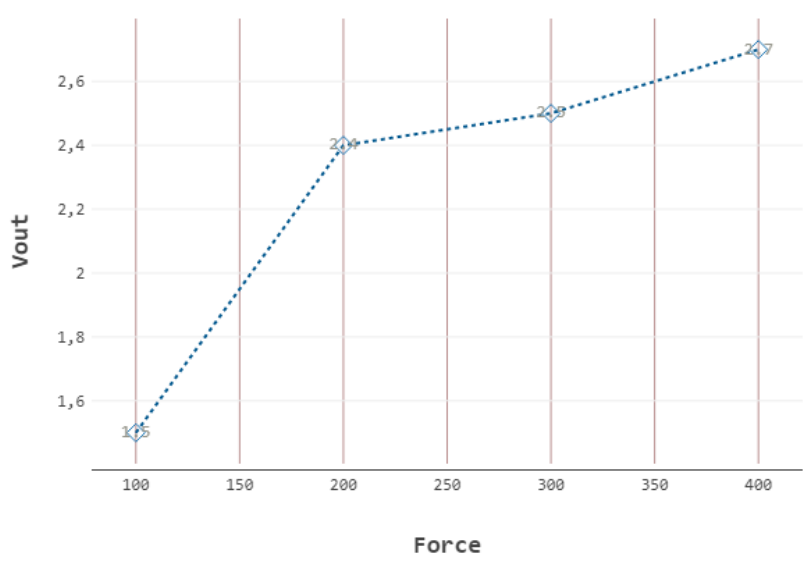

Fig. 12. Force Sensitivity Sensor.

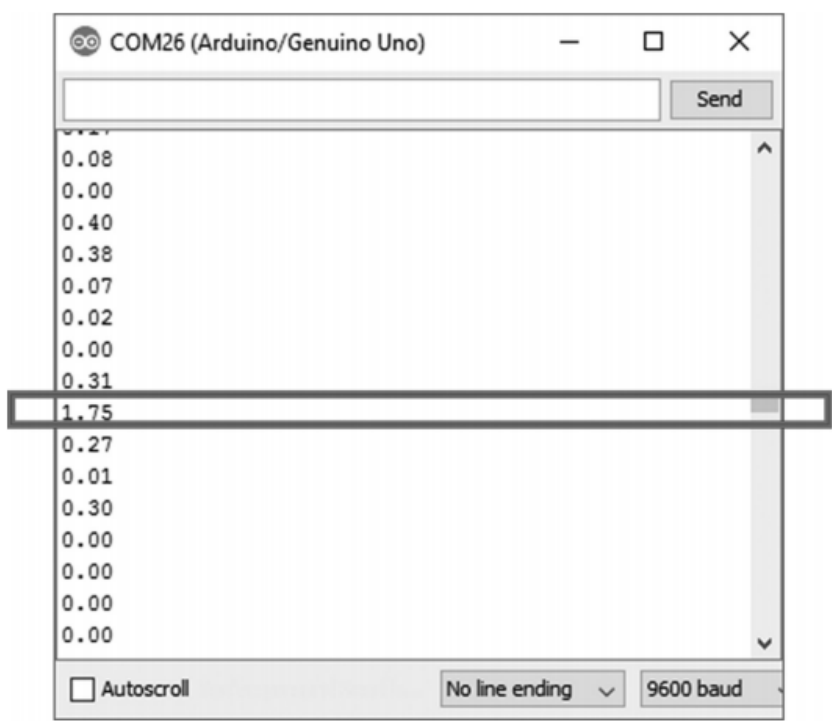

Fig. 13. Serial Monitor for Vibration Sensor.

\section{B. Final Output of the Research and Conclusion}

A competent Smart Vehicle Security and Safety System integrated with a pressure sensor, eye-blink sensor, alcohol

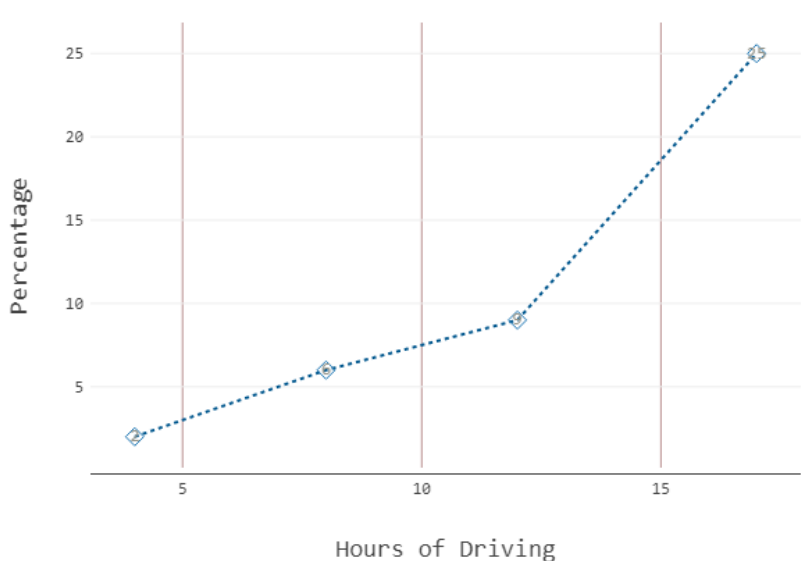

Fig. 14. Percentage of Crashes Due to Fatigue.

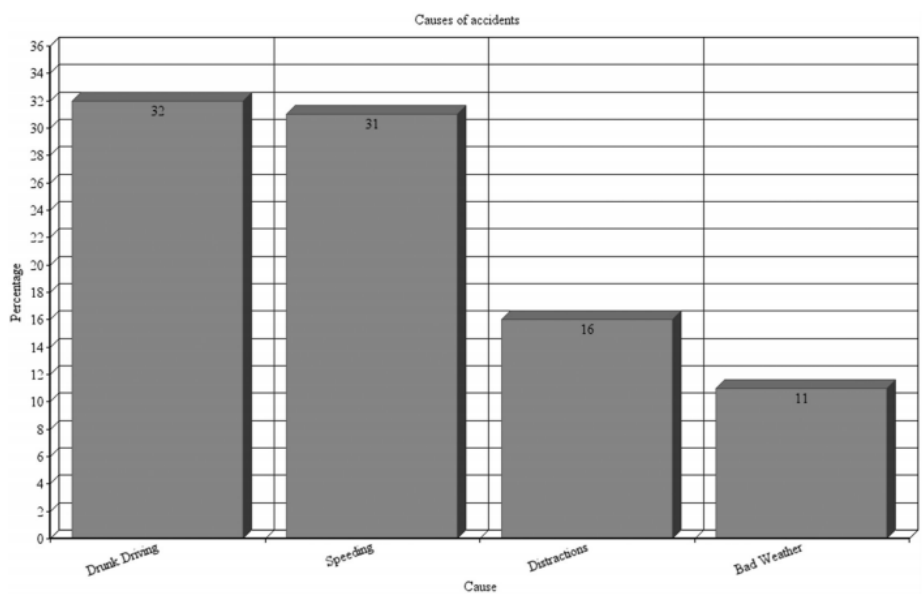

Fig. 15. Causes of Crashes.

sensor, proximity sensor, and vibration sensor (Figure 16) using the concept of GPS and GSM has been implemented. The sensors are integrated with the Arduino board. Areas with outreach problems that experience bad network connectivity or in remote areas with no network connectivity available can be an issue.

This can in turn lead to the accident intimidation text not being sent to the specified number. The proposed and thus implemented system can be enhanced and modified by adding concepts of technology such as big data and GPS to study the thus collected data to understand and read the patterns associated with the crashes. The same system can be modified accordingly and implemented for two Wheeler's. Further, the location of the crash can be sent to an ambulance as well for quick medical response and attention.

\section{REFERENCES}

[1] G. S. Pannu, M. D. Ansari, and P. Gupta, "'design and implementation of autonomous car using raspberry pi." international journal of computer applications 113," no., vol. 9, 2015 


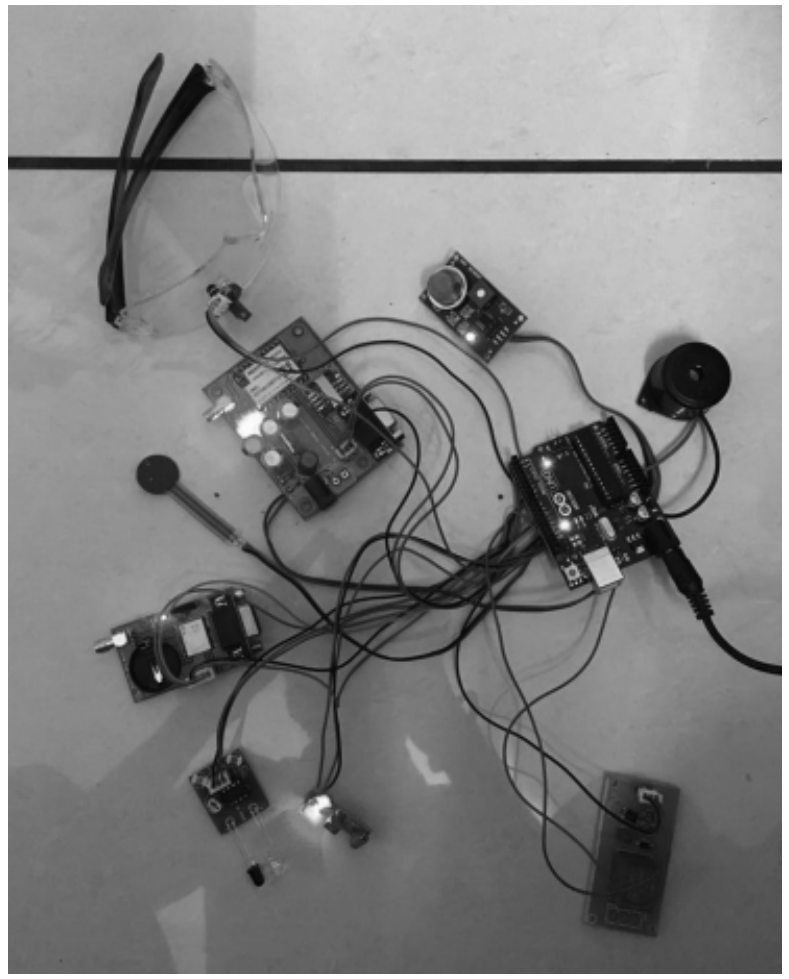

Fig. 16. Hardware Prototype.

[2] V. N. Kumar, V. S. Reddy, and L. P. Sree, “"'design and development of accelerometer based system for driver safety." international journal of science," Engineering and Technology Research (IJSETR), vol. 3, p. 12, 2014.

[3] C. Hahn, S. Feld, and H. Schroter, "Predictive collision management for time and risk dependent path planning," in Proceedings of the 28th International Conference on Advances in Geographic Information Systems, ser. SIGSPATIAL '20. New York, NY, USA: Association for Computing Machinery, 2020, p. 405-408. [Online]. Available: https://doi.org/10.1145/3397536.3422252

[4] G. N. A. H. Yar, A.-B. Noor-ul Hassan, and H. Siddiqui, "Real-time shallow water image retrieval and enhancement for low-cost unmanned underwater vehicle using raspberry pi," in Proceedings of the 36th Annual ACM Symposium on Applied Computing, ser. SAC '21. New York, NY, USA: Association for Computing Machinery, 2021, p. 1891-1899. [Online]. Available: https://doi.org/10.1145/3412841.3442060

[5] A. F. B. A. de Oliveira and L. V. L. Filgueiras, "Developer assistance tools for creating native mobile applications accessible to visually impaired people: A systematic review," in Proceedings of the 17th Brazilian Symposium on Human Factors in Computing Systems, ser. IHC 2018. New York, NY, USA: Association for Computing Machinery, 2018. [Online]. Available: https://doi.org/10.1145/3274192.3274208

[6] S. L. Fong, D. C. W. Yung, F. Y. H. Ahmed, and A. Jamal, "Smart city bus application with quick response (qr) code payment," ser. ICSCA '19. New York, NY, USA: Association for Computing Machinery, 2019, p. 248-252. [Online]. Available: https://doi.org/10.1145/3316615.3316718

[7] G. K. Gudur, A. Ramesh, and S. R, "A vision-based deep on-device intelligent bus stop recognition system," in Adjunct Proceedings of the 2019 ACM International Joint Conference on Pervasive and Ubiquitous Computing and Proceedings of the 2019 ACM International Symposium on Wearable Computers, ser. UbiComp/ISWC '19 Adjunct. New York, NY, USA: Association for Computing Machinery, 2019, p. 963-968. [Online]. Available: https://doi.org/10.1145/3341162.3349323

[8] D. Saha, M. Shinde, and S. Thadeshwar, "Iot based air quality monitoring system using wireless sensors deployed in public bus services," ser. ICC ,17. New York, NY, USA: Association for Computing Machinery, 2017. [Online]. Available: https://doi.org/10.1145/3018896.3025135

[9] M. Kumar, "R., and dr," R. Senthil. Effective control of accidents using routing and tracking system with integrated network of sensors, vol. 2, p. 4, 2013.

[10] R. Liu, Z. Yin, W. Jiang, and T. He, "Wibeacon: Expanding ble location-based services via wifi," in Proceedings of the 27th Annual International Conference on Mobile Computing and Networking, ser. MobiCom '21. New York, NY, USA: Association for Computing Machinery, 2021, p. 83-96. [Online]. Available: https://doi.org/10.1145/3447993.3448615

[11] J. J. T. Dai, X. Bai, and Z. Shen, "Mobile phone based drunk driving detection pervasive computing technologies for healthcare. 2010, 4th international ieee conference," $p$, vol. 1, March 2010.

[12] H. Chen, Y. Chiang, F. Chang, and H. Wang, "Toward real-time precise point positioning: Differential gps based on igs ultra rapid product. sice annual conference," The Grand Hotel, Taipei, Taiwan, August, vol. 18.

[13] X. Liu, X. Xu, X. Chen, E. Mai, H. Y. Noh, P. Zhang, and L. Zhang, "Individualized calibration of industrial-grade gas sensors in air quality sensing system," ser. SenSys '17. New York, NY, USA: Association for Computing Machinery, 2017. [Online]. Available: https://doi.org/10.1145/3131672.3136998

[14] A. T. Duchowski, S. Jörg, T. N. Allen, I. Giannopoulos, and K. Krejtz, "Eye movement synthesis," in Proceedings of the Ninth Biennial ACM Symposium on Eye Tracking Research \& Applications, ser. ETRA '16. New York, NY, USA: Association for Computing Machinery, 2016, p. 147-154. [Online]. Available: https://doi.org/10.1145/2857491.2857528 